\title{
EFICIENCIA DE VESTIMENTAS HIDRORREPELENTES NOVAS NA PROTEÇÃO DO TRATORISTA EM PULVERIZAÇÕES DE AGROTÓXICOS EM GOIABA COM O TURBOPULVERIZADOR ${ }^{1}$ MATHEUS BELLINI TÁCIO², MAURÍCIO LEITE DE OLIVEIRA ${ }^{3}$, JOAQUIM GONÇALVES MACHADO NETO
}

RESUMO-Os objetivos do trabalho foram: quantificar as exposições dérmicas e respiratórias potenciais proporcionadas ao tratorista em pulverizações de agrotóxicos, na cultura de goiaba, com o turbopulverizador; avaliar a eficiência de duas vestimentas de proteção individual; avaliar a segurança e classificar estas condições de trabalho em seguras ou inseguras para cada agrotóxico considerado. A exposição dérmica potencial proporcionada ao tratorista pela condição de trabalho foi de $3.807,3 \mathrm{~mL}$ de calda/7h. A eficiência do conjunto de proteção individual Agro Light foi de 96,7 \%, e a do Azeredo, 96,2 \%. A exposição dérmica potencial nas regiões do corpo mais expostas do tratorista à pulverização, em ordem decrescente, foram os pés, os braços, as coxas+pernas-frente e o tronco-atrás. A exposição dérmica não-controlada pelos conjuntos de proteção individual foi igualmente distribuída em todas as regiões do corpo do tratorista devido aos controles das exposições dérmicas que proporcionaram às regiões mais expostas do corpo. Foram classificadas como seguras as pulverizações de três dos 17 agrotóxicos considerados e sete com o uso das vestimentas de proteção individual. As demais foram classificadas como inseguras.

Termos para indexação: agrotóxicos, exposição dérmica, exposição respiratória, goiaba.

\section{EFFICIENCY OF NEW CLOTHES WATERREPELENT IN THE PROTECTION OF THE TRACTOR- DRIVER IN PESTICIDE SPRAYING IN GUAVA ORCHARDS WITH THE AIR-ASSISTED SPRAYER}

ABSTRACT-The objectives were to quantify potential dermal and respiratory exposure of the tractor-driver during spraying in guava orchards with the air-assisted sprayer, to evaluate the efficiency of two types of personal protective equipment (PPE), and to classify these working conditions as safe or unsafe for each pesticide studied. The potential dermal exposure of the tractor driver was $3,807.349 \mathrm{~mL}$ of spray $/ 6 \mathrm{~h}$. The efficiency in the control of dermal exposure was $96.7 \%$ for the Agro Light set and $96.2 \%$ for the Azeredo set. The feet, arms, thighs front legs and back trunk were the areas most exposed in the absence of protective measures, while exposure was generalized over the body when the PPE was used. The working conditions were considered to be safe in the case of 3 of the 17 pesticides tested in the absence of protective measures, and in the case of 4 additional pesticides when the Agro Light and Azeredo PPE was used, for a total of 7 pesticides.

Index Terms: pesticide, dermal exposure, respiratory exposure, guava.

\section{INTRODUÇÃO}

No Estado de São Paulo, encontram-se 57 \% da área cultivada com goiaba do País e 2.030 pequenos produtores, onde a maior produção de goiaba para a indústria se localiza nos municípios de Taquaritinga, Itápolis, Vista Alegre do Alto e Monte Alto (CATI, 2003). As aplicações dos agrotóxicos nas plantas de goiaba são realizadas com o turbopulverizador de arrasto ao trator, com pulverizações de altos volumes de caldas, resultando em grande deriva, que pode proporcionar elevadas exposições aos tratoristas. Portanto, nestas condições de trabalho, existem riscos de intoxicação do tratorista que devem ser avaliados e, se forem classificados como inaceitáveis, medidas de segurança devem ser implementadas.

As medidas de proteção atuam na redução da exposição nas vias de absorção dos agrotóxicos no corpo, que são a dérmica e a respiratória. Em diversos estudos realizados no País e no exterior, verifica-se que a quase totalidade da exposição dos trabalhadores aos agrotóxicos ocorre na via dérmica (Oliveira, 2000; Oliveira \& Machado Neto, 2003; Wolfe et al., 1972; WHO, 1982 e Van Hemmen, 1992).

De maneira geral, o uso de equipamentos de proteção individual (EPIs) é a única medida de proteção que se tem recomendado para o trabalho com agrotóxicos em condições de campo. Entretanto, os EPIs disponíveis no mercado proporcionam grande retenção de umidade e de calor na superfície do corpo, o que causa grande desconforto ao trabalhador e uso insuportável em condições de campo. Além do desconforto, a recomendação dos EPIs normalmente é realizada de forma errônea, o que agrava ainda mais o problema do uso dessas vestimentas. Os EPIs estão sendo recomendados apenas com base na classe toxicológica do agrotóxico, mas controlam as exposições dos trabalhadores, que são desconhecidas e desconsideradas.

${ }^{1}$ (Trabalho 074-07). Recebido em: 23-3-2007. Aceito para publicação em: 08-11-2007.Financiado pela FAPES

2Engenheiro Agrônomo- agrobuck@yahoo.com.br.

${ }^{3}$ Doutor em Agronomia e Engenheiro de Segurança do Trabalho. oliveiraml@hotmail.com.

${ }^{4}$ Prof. Dr. da FCAV/UNESP Câmpus de Jaboticabal - joaquim@fcav.unesp.br. 
A eficiência de materiais porosos e não-porosos ao inseticida carbaryl e ao herbicida atrazine foi avaliada por Raheel (1988). Os tecidos tratados com repelentes, em ordem decrescente de eficiência de proteção, foram: Tyvek ${ }^{\circledR}, 100 \%$ algodão, $65 / 35$ algodão/poliéster e 50/50 algodão/poliéster. A proteção do Tyvek ${ }^{\circledR}$ e do tecido com $100 \%$ de algodão com acabamento foi próxima de $100 \%$. A proteção dos tecidos confeccionados com as misturas de algodão/poliéster com acabamento repelente foi próxima de $99,5 \%$. Todos os tecidos tratados com repelente foram menos permeáveis aos agrotóxicos que os tecidos não-tratados. $\mathrm{O}$ poliéster afetou negativamente a proteção dos materiais.

Em alguns trabalhos realizados no País, foi avaliada a eficiência de conjuntos de proteção individual hidrorrepelentes na proteção dos aplicadores de agrotóxicos e está sendo avaliada com vestimentas novas, sem uso e lavagem para descontaminação, em diversas atividades de trabalho e culturas agrícolas. Oliveira (2000) avaliou a eficiência de um conjunto hidrorrepelente da marca AZR novo para atividade de tratorista, em aplicações de agrotóxicos em citros, com o turbopulverizador. O conjunto de proteção individual reduziu a exposição dérmica do tratorista em 96,7\%. Lapuente (1996) verificou que o conjunto AZR novo controlou $70 \%$ da exposição dérmica do tratorista em aplicações de agrotóxicos, em cultura de citros, com o turbopulverizador. Esse valor é inferior aos $96,7 \%$ avaliados por Oliveira (2000) nas mesmas condições de trabalho. Essa diferença pode ser devida à menor exposição dérmica proporcionada ao tratorista pelas condições de trabalho avaliadas por Lapuente (1996).

Em cultura de cana-de-açúcar, Momesso \& Machado Neto (2003) verificaram que o conjunto de proteção individual AZR ${ }^{\circledR}$ reduziu entre 49,2 e $85,2 \%$ a exposição dérmica potencial do tratorista aplicando herbicida com um pulverizador de barras montado em trator.

Em culturas de soja e amendoim, Cristóforo (2003) verificou que um conjunto de equipamento de proteção individual hidrorrepelente controlou $76,5 \%$ da exposição dérmica do tratorista aplicando herbicidas em pré-plantio incorporado ao solo e 50,9\% em pré-plantio, com um pulverizador de barra montado em trator. Nas pulverizações de inseticidas, aos 30 e 90 dias após a semeadura da soja, a eficiência do conjunto de EPIs foi de 75,3 e 86,3\%, respectivamente. Na cultura do amendoim, a eficiência foi de $83,3 \%$ na aplicação realizada aos 15 dias após a semeadura, $66,1 \%$ aos 60 e $77,4 \%$ aos 80 dias.

Portanto, há a necessidade de se quantificarem as exposições proporcionadas pelas condições específicas de trabalho e, juntamente com os dados toxicológicos específicos de cada agrotóxico, de se calcularem o risco de intoxicação e a segurança de cada condição de trabalho e, se necessário, de se recomendarem medidas de proteção para as condições inseguras. A toxicidade está disponível no conjunto dos dados toxicológicos dos agrotóxicos, mas a exposição ocupacional tem de ser quantificada nas condições específicas de trabalho. Com esses dados, calcula-se a margem de segurança (MS) para classificar as condições de trabalho em seguras ou inseguras.

Com o valor da MS, calcula-se o tempo de trabalho seguro (TTS) para determinar a intensidade da segurança para cada agrotóxico que pode ser aplicado ou a condição de trabalho. Para as condições classificadas como inseguras, calcula-se a necessidade de controle das exposições (NCE) para torná-las seguras com a medida de segurança mais adequada em termos de eficiência, economia e conforto para o trabalhador. O controle da toxicidade é realizado pela substituição do agrotóxico por outro menos tóxico (com maior valor de NOEL). O NOEL é a dose que não causa efeitos observados em animais de laboratório alimentados com rações contaminadas com o produto químico em estudo. Porém, essa possibilidade é viável apenas se a eficiência agronômica do tratamento fitossanitário não for reduzida. A exposição pode ser controlada com medidas de proteção coletiva, aplicadas no ambiente e nos equipamentos de trabalho, ou individuais, que é o uso de EPls.

$\mathrm{Na}$ busca bibliográfica realizada, não foi encontrado nenhum trabalho sobre a avaliação do risco ou da segurança das condições de trabalho com agrotóxicos, na cultura de goiaba, no País. Entretanto, há a imposição legal para os empregadores realizarem avaliações dos riscos ocupacionais e de proporcionar condições de segurança aos trabalhadores com os agrotóxicos, de acordo com a NR 31, anexada à Portaria n. 86, de 04-03-2005 (BRASIL, 2005).

Os objetivos deste trabalho foram: quantificar as exposições dérmicas e respiratórias potenciais proporcionadas ao tratorista ao pulverizar agrotóxicos na cultura de goiaba com o turbopulverizador de arrasto; avaliar as exposições dérmicas nãocontroladas e a eficiência de dois conjuntos de proteção individuais confeccionados com tecido hidrorrepelente no controle da exposição dérmica do tratorista, em pulverizações de agrotóxicos, com o turbopulverizador de arrasto; classificar essas condições de trabalho com os conjuntos de proteção individual em seguras ou inseguras, para 17 agrotóxicos recomendados na cultura; calcular o TTS para os 17 agrotóxicos considerados e as NCEs para os agrotóxicos cujas condições de trabalho foram classificadas como inseguras $(\mathrm{MS}<1)$.

\section{MATERIAL E MÉTODOS}

As avaliações das exposições dérmicas e respiratórias potenciais e as exposições dérmicas não-controladas pelos dois conjuntos de proteção individual hidrorrepelentes, proporcionadas ao tratorista nas pulverizações de 17 agrotóxicos, foram realizadas em culturas de goiaba, no Município de Vista Alegre do Alto-SP.

As exposições do tratorista foram avaliadas em pulverizações com o turbopulverizador da marca Jacto, modelo Arbus, com tanque de $2.000 \mathrm{~L}$ e 26 bicos, 13 de cada lado da turbina de ar e pressão de trabalho entre 11 e $13 \mathrm{kgf} / \mathrm{cm}^{2}$. As pontas de pulverização utilizadas foram do modelo $\mathrm{J}-5$, sendo treze pontas de cada lado do pulverizador. Dessas pontas, oito estavam compostas com difusores de três furos, e cinco com difusores de dois furos. As plantas da cultura estavam, em média, com 2,5 $\mathrm{m}$ de altura e $6,5 \mathrm{~m}$ de diâmetro das copas, espaçadas em $7,5 \mathrm{~m}$ nas entrelinhas e $5 \mathrm{~m}$ entre plantas na linha de plantio. $\mathrm{O}$ trator utilizado foi um MF 275 (sem cabina e sem capota), trabalhando em $2^{\mathrm{a}}$ reduzida e na rotação de $1.600 \mathrm{rpm}$. O volume 
de calda aplicado variou entre 9,5 e $10 \mathrm{~L}$ por planta, pulverizando entre 200 e 210 plantas por tanque de calda.

Como medidas de proteção individual do tratorista, foram avaliados os conjuntos de proteção individual hidrorrepelentes Agro Light, produzido pela empresa R\&B equipamentos de segurança, e o denominado Kit Tratorizado, produzido pela Azeredo EPIs. Os dois conjuntos foram complementados com botas de borracha e máscara descartável com filtro de carvão ativado.

Para as avaliações das exposições dérmicas e respiratórias, foi utilizado o cátion Mn do sulfato de manganês como traçador nas caldas pulverizadas, de acordo com Oliveira \& Machado Neto (2003), adicionando-se às caldas de pulverização o sulfato de manganês ( $31 \%$ de Mn), na dosagem de $250 \mathrm{~g}$ do produto por 100 L de calda.

As exposições dérmicas foram avaliadas com 10 repetições, e o tratorista usava as seguintes vestimentas amostradoras e coletores/amostradores: macacões de mangas compridas com capuz, de tecido de brim, para quantificar as exposições dérmicas da cabeça + pescoço, tronco, braços e pernas; luvas de algodão, para quantificar a exposição dérmica das mãos; absorventes higiênicos femininos (Carefree), afixados sobre máscaras descartáveis semifaciais, para quantificar a exposição dérmica da face, e sobre botas de borracha na parte mediana superior dos pés, para quantificar as exposições dos pés.

As exposições respiratórias foram avaliadas com bombas pessoais com fluxo de ar contínuo, da marca A.P. Buck, reguladas para succionar $2 \mathrm{~L}$ de ar/min, de acordo com a metodologia adaptada por Oliveira e Machado Neto (2003). Cada bomba foi equipada com uma mangueira plástica contendo um cassete de acrílico na extremidade. Os cassetes continham filtros de éster celulose, com porosidade de $0,8 \mathrm{~mm}$ e diâmetro de $37 \mathrm{~mm}$, apoiados sobre suportes de papel de igual diâmetro.

Imediatamente após os períodos de exposição, as partes seccionadas dos macacões e os demais amostradores/coletores e os cassetes foram retirados do trabalhador e acondicionados em sacos plásticos, devidamente identificados. Essas amostras foram levadas ao laboratório para a quantificação do cátion $\mathrm{Mn}$ capturado, de acordo com Oliveira e Machado Neto (2003). A solubilização do cátion nas partes seccionadas dos macacões, nos coletores e nas membranas de éster celulose, foi realizada com uma solução contendo $\mathrm{HCl}$ 0,2 N. As concentrações do traçador nos extratos, filtrados em papel de filtro qualitativo, foram quantificadas na chama de um espectrofotômetro de absorção atômica, com lâmpada, para a leitura de manganês. As exposições dérmicas e respiratórias às caldas foram calculadas utilizando-se dos dados de quantidade do cátion Mn recuperada de cada amostrador/coletor utilizado e da concentração do traçador nas respectivas caldas pulverizadas a que os tratoristas ficaram expostos durante o tempo de amostragem.

A eficiência dos conjuntos hidrorrepelentes de proteção individual avaliados foi calculada por meio da redução percentual da exposição dérmica potencial avaliada em relação à exposição dérmica não-controlada (EDNC) pelos mesmos. A eficiência da máscara descartável com filtro de carvão ativado e de botas de borracha impermeáveis, que completaram os conjuntos de EPIs, foi considerada como $95 \%$ (Lundehn et al., 1992).

A segurança do tratorista na pulverização de cada um dos 17 agrotóxicos considerados foi classificada com base no cálculo da margem de segurança (MS), com a fórmula proposta por Severn (1984), que é a seguinte:

$$
\mathrm{MS}=\frac{\text { NOEL } \times \text { P }}{\text { QAE } \times 10}
$$

Onde: NOEL = nível de efeitos não-observados (mg/kg/dia) publicados pela TGA (2003); P = peso corpóreo médio do trabalhador, considerado como $70 \mathrm{~kg}$, e $\mathrm{QAE}=$ quantidade absorvível da exposição ( $\mathrm{mg} / \mathrm{kg} / \mathrm{dia})$.

A quantidade absorvível da exposição na via dérmica foi considerada como sendo $10 \%$ da exposição dérmica avaliada, segundo Feldman e Maibach (1974), citados por Byers et al. (1992), e $100 \%$ da exposição respiratória avaliada. $\mathrm{O}$ valor 10 no denominador, multiplicado pela quantidade absorvível, é o fator de segurança utilizado para compensar a extrapolação dos valores de NOEL, obtido em animal de laboratório, para o homem (Brouwer et al., 1990).

A segurança das 17 condições de trabalho foi classificada com base no seguinte critério:

- se MS $\geq 1$, a condição de trabalho é classificada como segura, a exposição como tolerável e o risco de intoxicação, aceitável. Conseqüentemente, nessas condições, não há necessidade de se utilizar qualquer medida de proteção.

- se MS < 1, a condição de trabalho é classificada como insegura, a exposição como intolerável e o risco, inaceitável. Nas condições inseguras, há necessidade de se utilizarem medidas de segurança em intensidade suficiente para torná-las seguras, com pelo menos $\mathrm{MS}=1$.

Para as condições de trabalho classificadas como inseguras, foi calculada a necessidade de controle da exposição (NCE) para torná-las seguras, com a fórmula proposta por Machado Neto (1997), que é a seguinte:

$$
\mathrm{NCE}=\left(1-\mathrm{MS}_{<1}\right) \times 100(\%)
$$

Para qualquer condição de trabalho, o tempo de trabalho seguro (TTS) pode ser calculado e analisado como uma possibilidade de medida de segurança coletiva a ser utilizada. $\mathrm{O}$ TTS é o tempo a partir do início da atividade em que a exposição ao agrotóxico proporciona MS $\geq 1$. O TTS foi calculado para todas as condições de trabalho avaliadas, com a fórmula proposta por Machado Neto (1997), que é a seguinte:

$$
\text { TTS }=\text { MS } x \text { tee }
$$

Onde tee $=$ tempo de exposição efetiva, considerado em $7 \mathrm{~h}$.

A interpretação do valor do TTS é a seguinte: para as condições de trabalho segura, observa-se a intensidade da segurança em função do tempo de exposição e, para as inseguras, avalia-se se o TTS pode ser utilizado como medida de segurança.

\section{RESULTADOS E DISCUSSÃO}

As exposições dérmicas proporcionadas ao tratorista em aplicações de agrotóxicos, na cultura de goiaba, com o turbopulverizador, foram drasticamente superiores às respiratórias (Tabela 1). Esse resultado é similar a outros avaliados em diversas 
culturas agrícolas no Brasil (Oliveira, 2000; Oliveira \& Machado Neto, 2003) e no exterior (Wolfe et al., 1972; WHO, 1982 e Van Hemmen, 1992).

A exposição dérmica potencial proporcionada ao tratorista por essa atividade foi de $3.807,3 \mathrm{~mL}$ de calda/dia, e a respiratória, de apenas $0,024 \mathrm{~mL}$ de calda/dia, ou seja, $0,00063 \%$ da exposição total (Tabela 1). A exposição não-controlada pelo conjunto de proteção individual Agro Light foi de $123,9 \mathrm{~mL}$ de calda/dia, e pelo Kit Tratorizado da Azeredo, 144,3 mL. O conjunto de proteção individual da marca Agro Light controlou 96,7 \% da exposição dérmica potencial, e o da Azeredo EPI, 96,2\%.

Verifica-se que os controles da exposição dérmica do tratorista pulverizando agrotóxicos na cultura da goiaba, proporcionados pelos conjuntos Agro Light e Azeredo, foram próximos dos $96,7 \%$ avaliados por Oliveira (2000) com o conjunto hidrorrepelente da marca AZR. As eficiências avaliadas neste trabalho foram superiores aos $70 \%$ avaliados por Lapuente (1996) com o conjunto AZR para o tratorista, em aplicações de agrotóxicos, em cultura de citros, com o turbopulverizador, aos diversos valores avaliados por Cristóforo (2003), em culturas de soja e amendoim. Portanto, as eficiências avaliadas por Lapuente (1996) e Cristóforo (2003) são inferiores às avaliadas neste trabalho e no de Oliveira (2000).

Para as condições de trabalho classificadas como inseguras, os dois conjuntos de equipamentos de proteção individual foram eficientes para tornar seguras condições de trabalho inseguras cujas NCEs calculadas foram inferiores a 96,7\% com o Agro Light e 96,2\% com o da marca Azeredo EPI. Portanto, os dois conjuntos de proteção individual foram eficientes para tornar segura para o tratorista apenas nas pulverizações de tiofanato metílico e deltametrina, e dos fungicidas triadimenol e triforine.

Nos valores do TTS calculados, observa-se a intensidade da segurança das condições de trabalho para a atividade de tratorista na aplicação de agrotóxicos na cultura de goiaba. $\mathrm{O}$ TTS não pode ser utilizado como medida de segurança coletiva na condição de trabalho sem proteção. Isto porque o uso do TTS como medida de segurança somente é viável na prática e economicamente para condições de trabalho com valores de TTS ligeiramente superiores a meia jornada de trabalho. Neste caso, o trabalhador aplica agrotóxicos durante meia jornada diária e recebe apenas a metade da exposição total da jornada de trabalho diário. No resto do dia, vai realizar outro tipo de atividade sem exposição a agrotóxicos.

Com o uso dos conjuntos de proteção, o TTS pode ser usado como medida de segurança apenas na pulverização de clorotalonil. Os tempos de exposição devem ser reduzidos para 4,23 h na pulverização deste fungicida com o uso do conjunto Agro Light e, em 3,63 h, com o da Azeredo EPI.

A exposição dérmica sofrida pelo tratorista aplicando agrotóxicos com o turbopulverizador, na cultura de goiaba (3.807,3 $\mathrm{mL}$ de calda/dia), foi drasticamente superior aos $300 \mathrm{~mL}$ de calda/ dia relatados por Suzuki (1991), aos $60 \mathrm{~mL}$ de calda/dia relatados por Lapuente (1996) e aos 217,3 e 496,8 $\mathrm{mL}$ de calda/dia relatados por Oliveira (2000), todos na cultura de citros. As grandes diferenças nos valores das exposições dérmicas sofridas pelo tratorista, avaliadas nestes trabalhos, podem ser explicadas pelas diferenças dos tratores. Nas avaliações de Lapuente (1996) e de Oliveira (2000), os tratores estavam equipados com a capota original, que cobria a região superior (teto) do banco do tratorista. O trator utilizado nas avaliações realizadas no presente trabalho estava desprovido de capota. Portanto, a ausência da capota resultou em maiores exposições dérmicas dos tratoristas, e, assim, a própria capota original do trator pode comportar-se também como uma medida de proteção coletiva, cuja eficiência deve ser avaliada separadamente.

A exposição dérmica potencial do tratorista foi concentrada nos membros inferiores e superiores (Figura 1). Em ordem decrescente, as regiões mais atingidas foram os pés, que receberam $35,6 \%$ da exposição total, os braços $(13,5 \%)$, as coxas+pernas-frente $(12,1 \%)$ e o tronco-atrás $(10,6 \%)$. Essa distribuição da exposição dérmica potencial no corpo do tratorista, sem medidas de proteção, em pulverizações de agrotóxicos, na cultura da goiaba, diferiu de outras avaliadas em trabalhos encontrados na literatura, em cultura de citros, com o mesmo tipo de pulverizador (Lapuente, 1996 e Oliveira, 2000). Esse fato pode ser explicado pela variação das condições climáticas no momento das avaliações, principalmente a velocidade e a direção do vento, e o modelo do trator.

Com a utilização dos EPIs Agro Light e Azeredo, verificase que a distribuição da exposição dérmica não-controlada foi generalizada e com valores mais próximos nas várias regiões do corpo do tratorista (Figura 1). Esta distribuição da exposição dérmica nas regiões do corpo do tratorista usando conjuntos de EPIs hidrorrepelentes é semelhante à avaliada por Oliveira (2000) em aplicações de agrotóxicos, em cultura de citros, com esse mesmo tipo de pulverizador.

De acordo com os valores da margem de segurança (MS) calculados sem o uso dos conjuntos de EPIs, foram classificadas como condições de trabalho seguras ( $\mathrm{MS}^{3} 1$ ), para o tratorista, as pulverizações dos agrotóxicos azoxystrobin, betacyflutrin e cyproconazole; e para o tratorista, com uso dos dois conjuntos de EPIs Agro Light e Azeredo, também passaram a ser classificadas como seguras, além das três pulverizações seguras sem as vestimentas de proteção, as de deltametrina, tiofanato metílico, triadimenol e triforine, conforme se observa na Tabela 2. Para os demais agrotóxicos considerados, as condições de trabalho foram classificadas como inseguras, e há a necessidade de se estudar o uso de outras medidas de segurança ou não recomendá-las nestas condições de trabalho avaliadas.

TABELA 1- Exposições dérmica e respiratória, potenciais (tratorista sem EPI) e não-controladas (tratorista com EPI) pelos EPIs, sofridas pelo tratorista pulverizando agrotóxicos, na cultura da goiaba, com um turbopulverizador. (Vista Alegre do Alto $\mathrm{SP} / 2004)$

\begin{tabular}{|c|c|c|c|c|}
\hline \multirow{2}{*}{ ATIVIDADES } & \multicolumn{3}{|c|}{ Exposições (mL de calda/7 horas ) } & \multirow{2}{*}{$\begin{array}{c}\text { \% de } \\
\text { controle }\end{array}$} \\
\hline & ED & ER & Total (ED+ER) & \\
\hline Tratorista (EP) & $3.807,3$ & 0,024 & $3.807,4$ & - \\
\hline Tratorista com EPI Agro Light & 123,9 & $0,001 *$ & 123,9 & 96,7 \\
\hline Tratorista com EPI Azeredo & 144,3 & $0,001 *$ & 144,4 & 96,2 \\
\hline
\end{tabular}

Rev. Bras. Frutic., Jaboticabal - SP, v. 30, n. 1, p. 106-111, Março 2008 
TABELA 2 -Valores das margens de segurança (MS), necessidade de controle da exposição (NCE) e dos tempos de trabalho seguros (TTS), na atividade de tratorista aplicando os agrotóxicos registrados e não-registrados, na cultura da goiaba, com o turbopulverizador.

\begin{tabular}{|c|c|c|c|c|c|c|c|c|c|}
\hline \multirow{2}{*}{ Nome comum } & \multicolumn{3}{|c|}{ Sem Proteção } & \multicolumn{3}{|c|}{ EPI Agro Light } & \multicolumn{3}{|c|}{ EPI Azeredo } \\
\hline & MS & NCE & TTS & MS & NCE & TTS & MS & NCE & TTS \\
\hline acefato** $^{* *}$ & 0,01 & 99,5 & 0,04 & 0,17 & 83,5 & 1,16 & 0,14 & 85,8 & 0,99 \\
\hline azoxystrobin* & 2,30 & - & 16,09 & 70,48 & - & 493,38 & 60,54 & - & 423,79 \\
\hline betacyflutrin** & 3,68 & - & 25,74 & 112,77 & - & 789,41 & 96,87 & - & 678,06 \\
\hline & 0,02 & 98,0 & 0,14 & 0,60 & 39,6 & 4,23 & 0,52 & 48,1 & 3,63 \\
\hline cyproconazole* & 1,23 & - & 8,58 & 37,59 & - & 263,14 & 32,29 & - & 226,02 \\
\hline deltametrina** & 0,31 & 69,4 & 2,15 & 9,40 & - & 65,78 & 8,07 & - & 56,51 \\
\hline & 0,01 & 99,4 & 0,04 & 0,19 & 81,2 & 1,32 & 0,16 & 83,9 & 1,13 \\
\hline fenitrothion** & 0,01 & 99,5 & 0,03 & 0,15 & 85,0 & 1,05 & 0,13 & 87,1 & 0,90 \\
\hline 1 & 0,00 & 99,8 & 0,01 & 0,05 & 95,5 & 0,32 & 0,04 & 96,1 & 0,27 \\
\hline lambdacyhalothrin** & 0,01 & 99,1 & 0,06 & 0,28 & 71,8 & 1,97 & 0,24 & 75,8 & 1,70 \\
\hline methidathion** & 0,00 & 99,6 & 0,03 & 0,11 & 88,7 & 0,79 & 0,10 & 90,3 & 0,68 \\
\hline paration metílico** & 0,00 & 99,9 & 0,00 & 0,02 & 98,1 & 0,13 & 0,02 & 98,4 & 0,11 \\
\hline & 0,08 & 92,5 & 0,53 & 2,30 & - & 16,11 & 1,98 & - & 13,84 \\
\hline triadimenol** & 0,37 & 63,2 & 2,57 & 11,28 & - & 78,94 & 9,69 & - & 67,81 \\
\hline triclorfon* & 0,00 & 99,8 & 0,02 & 0,08 & 92,5 & 0,53 & 0,07 & 93,5 & 0,45 \\
\hline triforine** & 0,17 & 82,6 & 1,22 & 5,34 & - & 37,39 & 4,59 & - & 32,12 \\
\hline
\end{tabular}

TTS foi calculado para o tempo de exposição efetivo de 7 horas. *produtos registrados. **produtos não-registrados.

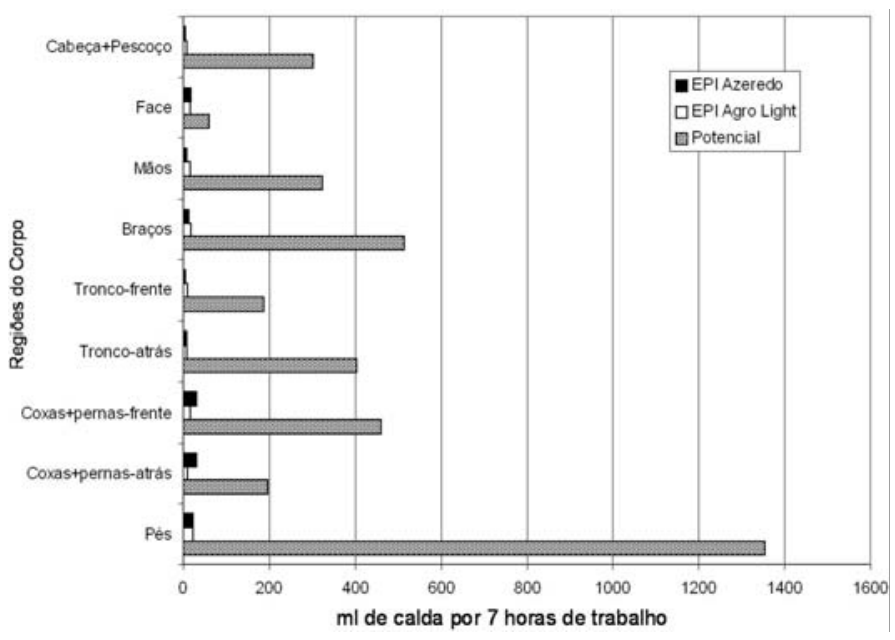

FIGURA 1 - Distribuição das exposições dérmicas em mL de calda por 7 horas de trabalho e percentual nas diversas regiões do corpo do tratorista, com EPI (exposição não-controlada pelo EPI) e sem EPIs (exposição potencial), em pulverizações de agrotóxicos, na cultura de goiaba, com o turbopulverizador.

\section{CONCLUSÕES}

1-Com os resultados obtidos nas condições de trabalho das atividades avaliadas, conclui-se que a pulverização dos agrotóxicos considerados, em cultura de goiaba, com o turbopulverizador, proporciona exposições aceitáveis ao tratorista para apenas três $(18,75 \%)$ dos dezessete agrotóxicos estudados.

2-O conjunto de proteção individual hidrorrepelente Agro Light, produzido pela empresa $R \& B$ equipamentos de segurança, e o denominado Kit Tratorizado, produzido pela Azeredo, controlam a exposição proporcionada ao tratorista o suficiente para tornar seguras as pulverizações de sete $(43,75 \%)$ dos dezessete agrotóxicos considerados. As regiões do corpo do tratorista, sem medidas de proteção, mais expostas são os pés, os braços, a frente das coxas e pernas, e atrás do tronco.

\section{REFERÊNCIAS}

BRASIL. Ministério do Trabalho. Norma Regulamentadora de Segurança e Saúde no Trabalho na Agricultura, Pecuária, Silvicultura, Exploração Florestal e Aqüicultura - NR 31, Portaria n.86, 03 mar. 2005. Brasília: Diário Oficial da União, 2005. $30 \mathrm{p}$.

BROUWER, D. H.; BROUWER, R.; DE VREEDE, J.A.F.; DEMIK, G; VAN HEMMEN, J.J. Respiratory exposure to field-strength dusts in greenhouses during application and after re-entry. The Hague: TNO Health Research, 1990. p.183-184. (Annual Report).

BYERS, M. E.; KAMBLE, S. T.; WITKOWSKI, J. F.; ECHTENKAMS, G. Exposure of a mixer-loader to insecticides applied to Corn via a center-pivot irrigation system. Bulletin Environmental Contamination Toxicology, New York, v.49, p.58-65, 1992.

CATI. Coordenadoria de Assistência Técnica Integral. Produção integrada de frutas: goiaba. Disponível em: <URL: http:// www.cati.sp.gov.br>. Acesso em: 09 abr. 2003.

CRISTÓFORO, A.B. Segurança das condições de aplicação de agrotóxicos em cultura de soja e amendoim e eficiência de medidas de segurança individual e coletiva. 2003. 60f. Dissertação (Mestrado em Agronomia Área de Concentração em Entomologia Agrícola) - Faculdade de Ciências Agrárias e Veterinárias, Universidade Estadual Paulista, Jaboticabal, 2003.

LAPUENTE, D.B.G. Segurança das condições de aplicação de agrotóxico na cultura de citros.[Citrus sinensis (L.) Osbeck]. 1996. 47p. (Trabalho de Graduação em Agronomia) - Faculdade de Ciências Agrárias e Veterinárias, Universidade Estadual Paulista, Jaboticabal, 1996.

LUNDEHN. J.; WESTPHAL, D.; KIECZKA, H.; KREBS, B.; LÖCHER-BOLZ, S.; MAASFELD, W.; PICK, E.D. Uniform principles for safeguarding the health of applicators of plant protection products (Uniform principles for operator protection). Berlin: Kommissionsverlag Paul Parey, 1992. 90p. 
MACHADONETO, J.G. Proteção do aplicador de herbicidas. In: $21^{\circ}$ CONGRESSO BRASILEIRO DA CIÊNCIA DAS PLANTAS DANINHAS, 21., Caxumbu,MG, 1997. Palestras e mesas-redondas... p.135-144.

MOMESSO, J. C.; MACHADO NETO, J. G. Efeitos do período e volume de aplicação na segurança dos tratoristas aplicando herbicidas na cultura de cana-de-açúcar (Saccharum spp.). Planta Daninha, Viçosa, v.21, n.3, p.467-478, 2003.

OLIVEIRA, M.L. Segurança no trabalho de aplicação de agrotóxicos com turbopulverizador e pulverizador de pistolas em citros, na região de Jaboticabal- SP. 2000.99 f. Dissertação (Mestrado em Agronomia) - Faculdade de Ciências Agrárias e Veterinárias, Universidade Estadual Paulista., Jaboticabal, 2000.

OLIVEIRA, M. L.; MACHADO NETO, J. G. Use of manganese as tracer in the determination of respiratory exposure and relative importance of exposure routes in the safety of pesticide applicators in citrus orchards. Bulletin of Environmental Contamination Toxicology, New York, v.70, p.415-421, 2003.

RAHEEL, M. Dermal exposure to pesticides: the barrier effectiveness of protective clothing. Journal of Environmental Health, Abingdon, v.51, n.2, p.82-84, 1988.
SEVERN, D.J. Use of exposure data for risk assessment. In: SIEWIERSKI, M. (Ed). Determination and assessment of pesticide exposure. New York: Elsevier, 1984.p.13-19. (Studies in Environment Science, 24).

SUZUKI, R.T. Exposição dérmica e risco de intoxicação ocupacional de aplicadores de agrotóxicos em cultura de citros [Citrus sinensis (L.) Osbeck]. 1991.45 f. Monografia (Graduação em Agronomia) - Faculdade de Ciências Agrárias e Veterinárias de Jaboticabal, Universidade Estadual Paulista, Jaboticabal, 1991. TGA. Therapeutic goods administration. Ad List: Accept daily intakes for agricultural and veterinary chemicals. Australia.2003.

VAN HEMMEN, J.J. Agricultural pesticide exposure data bases for risk assessment. Reviews of Environmental Contamination and Toxicology, v. 126, p.85, 1992.

WHO - World Health Organization. Field surveys of exposure to pesticide-standard protocol. Geneva: WHO, 1982. (Document VBC/ 82.1).

WOLFE, H.R.; ARMSTRONG, J.F.; STAIFF, D.C.; COMER, S.W. Exposure of spraymen to pesticide. Archives Environ.mental Health, Washington, v. 25, p. 29-31, 1972. 